University of Nebraska - Lincoln

DigitalCommons@University of Nebraska - Lincoln

4-2004

\title{
Holographic recording of laser-induced plasma
}

\author{
Martin Centurion \\ University of Nebraska-Lincoln, martin.centurion@unl.edu \\ Ye Pu \\ California Institute of Technology, yepu@sunoptics.caltech.edu \\ Zhiwen Liu \\ California Institute of Technology \\ Demetri Psaltis \\ California Institute of Technology \\ Theodor W. Hänsch \\ Max-Planck-Institut für Quantenoptik,
}

Follow this and additional works at: https://digitalcommons.unl.edu/physicscenturion

Part of the Physics Commons

Centurion, Martin; Pu, Ye; Liu, Zhiwen; Psaltis, Demetri; and Hänsch, Theodor W., "Holographic recording of laser-induced plasma" (2004). Martin Centurion Publications. 6.

https://digitalcommons.unl.edu/physicscenturion/6

This Article is brought to you for free and open access by the Research Papers in Physics and Astronomy at DigitalCommons@University of Nebraska - Lincoln. It has been accepted for inclusion in Martin Centurion Publications by an authorized administrator of DigitalCommons@University of Nebraska - Lincoln. 


\title{
Holographic recording of laser-induced plasma
}

\author{
Martin Centurion, Ye Pu, Zhiwen Liu, and Demetri Psaltis \\ Department of Electrical Engineering, California Institute of Technology, Pasadena, California 91125
}

Theodor W. Hänsch

Max-Planck-Institut für Quantenoptik, D-85748 Garching, Germany

Received October 6, 2003

\begin{abstract}
We report on a holographic probing technique that allows for measurement of free-electron distribution with fine spatial detail. Plasma is generated by focusing a femtosecond pulse in air. We also demonstrate the capability of the holographic technique of capturing the time evolution of the plasma-generation process. (C) 2004 Optical Society of America

OCIS codes: $\quad 090.0090,090.4220,190.5530,190.7110,140.3440,320.2250$.
\end{abstract}

We describe the use of on-axis femtosecond holography to capture fast dynamic phenomena on a CCD camera. $^{1-4}$ The femtosecond laser allows us to sample very quickly changing events with 150 -fs resolution while holography makes it possible to accurately reconstruct the phase and amplitude of the recorded object. The on-axis holograms recorded on the CCD camera provide spatial resolution of approximately $4 \mu \mathrm{m}$. We have demonstrated this technique by recording holograms of the plasma trail generated by a pump femtosecond laser pulse propagating through air. The spatial distribution of the plasma density is retrieved from the measured phase profile. Propagation of short laser pulses in air has been a subject of intense interest, ${ }^{5-14}$ and several methods have been described for imaging the spatial distribution of the plasma trail created by laser pulses. ${ }^{8-11}$ The technique that we describe provides finer spatial resolution than previous methods and allows for the recording of a sequence of frames capturing the evolution of the event over time.

On-axis digital holograms are recorded with the setup in Fig. 1. We use a Ti:sapphire laser amplifier operating at $800-\mathrm{nm}$ wavelength to generate the ultrafast event and also to record it. The energy and the duration of the laser pulse are $2 \mathrm{~mJ}$ and $150 \mathrm{fs}$ FWHM, respectively. The pulse is split in two, with a major portion of the energy $(1.5 \mathrm{~mJ})$ going into the pump beam. After a variable delay, the pump beam is focused with an achromatic lens (L3) of 5-cm focal length. The peak power of the pump pulse is $10 \mathrm{GW}$, approximately six times larger than the critical power of $1.8 \mathrm{GW}$ for self-focusing in air. The probe pulse propagates in a direction perpendicular to the pump and captures the plasma trail of the pump pulse in the air. The image is magnified by a factor of $M=f_{2} / f_{1}$. We record an in-line (Gabor) hologram ${ }^{15}$ with the CCD camera at a distance $L$ from the image plane. The in-line hologram is essentially the interference between the diffraction of the plasma distribution and the probe beam. The hologram is then digitally reconstructed to retrieve the phase change induced in the probe by the plasma.

The phase of the object wave recovered through in-line holography is subject to errors due to artifacts surrounding the reconstructed object. We have performed computer simulations in which we digitally propagated a plane wave of $\lambda=800 \mathrm{~nm}$ through a one-dimensional Gaussian phase object along the center line for $30 \mathrm{~cm}$ to form a hologram. The waist sizes of the objects are 70 and $350 \mu \mathrm{m}$, covering approximately $7 \%$ and $35 \%$ of the hologram area, respectively. The peak phase change of both objects is $-0.8 \mathrm{rad}$. The phase change induced by the object is then retrieved through numerical reconstruction and compared with the original phase distribution. The phase error for a $70-\mu \mathrm{m}$ object is approximately $6 \%$ and is greater than $50 \%$ for a $350-\mu \mathrm{m}$ object. In general, in-line holography is unable to retrieve the phase for large, strongly diffracting objects, since it becomes very difficult to separate the real image from the virtual image. In our experiment the overall lateral size of the object is roughly $60 \mu \mathrm{m}$, which covers no more than $7.5 \%$ of the hologram after a magnification of 12.5. Therefore, we estimate that the error in the phase change retrieved from the digital reconstruction

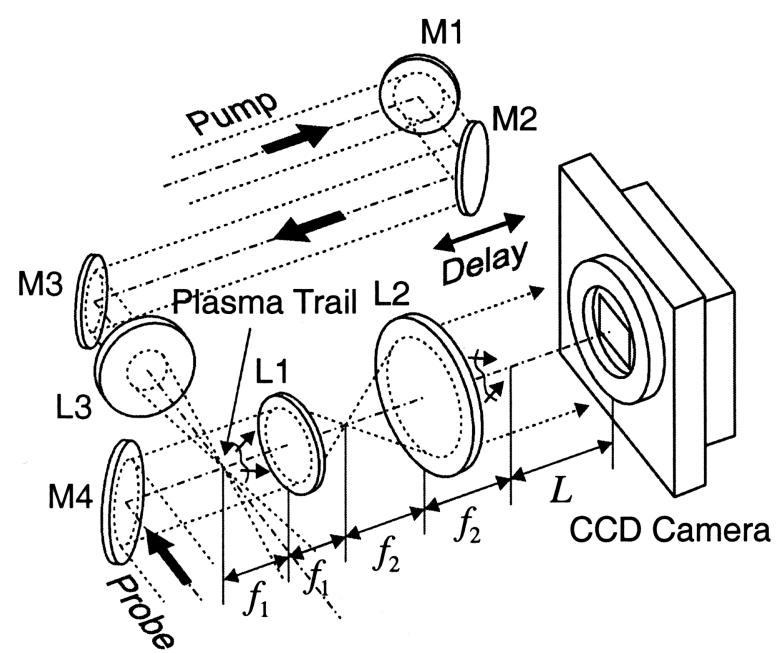

Fig. 1. Holographic setup. An intense femtosecond laser pulse (the pump pulse) is focused by L3 and leaves a plasma trail. A probe pulse traverses the plasma trail a few picoseconds after the pump pulse goes through. The system has a magnification of $f_{2} / f_{1}$. L $1-\mathrm{L} 3$, lenses; M1-M4, mirrors. 
is less than 6\%. Figure 2(a) shows the hologram captured by the CCD camera. The pulse propagates from left to right. A separate image frame of the probe beam alone is subtracted from the hologram to remove spatial nonuniformity. We numerically backpropagate this hologram to reconstruct the object wave front and retrieve the phase change due to the object (the plasma distribution). The reconstruction algorithm calculates the convolution between the holographic fringes and a Fresnel phase kernel. ${ }^{16}$

Ignoring diffraction through the $60-\mu \mathrm{m}$ object, we find that the phase change $\Delta \varphi$ that the probe pulse undergoes after passing through the plasma is $\Delta \varphi(x, y)=\int_{0}^{s} 2 \pi \Delta n(x, y, z) / \lambda \mathrm{d} z$, where $s$ is the size of the plasma and $\lambda$ is the wavelength. It is in general difficult to calculate the corresponding index change if $\Delta n$ contains three-dimensional structures. This change of index is related to the density of free electrons as ${ }^{17} n_{0}+\Delta n=\epsilon^{1 / 2}=\left(1-\omega_{p}^{2} / \omega^{2}\right)^{1 / 2}$, where $n_{0}$ is the refractive index of air, $\epsilon$ is the electrical permittivity, $\omega$ is the frequency of the probe pulse, and $\omega_{p}$ is the plasma frequency. From this relationship we obtain the density of free electrons as $n_{e}=-2 \Delta n n_{\mathrm{cr}}$, where $n_{\text {cr }}=1.7 \times 10^{21} \mathrm{~cm}^{-3}$ is the critical plasma density at the 800-nm wavelength. Using plasma size $s=60 \mu \mathrm{m}$, we estimate that the maximum index change is approximately $-2.12 \times 10^{-3}$, corresponding to a free-electron density of $7.03 \times 10^{18} \mathrm{~cm}^{-3}$. On the other hand, the mean electron density over the $60-\mu \mathrm{m}$ cross section is $5.53 \times 10^{17} \mathrm{~cm}^{-3}$.

We show the spatial map of free-electron density in Fig. 2(b). The initial disturbance splits into several branches after propagating for $100 \mu \mathrm{m}$ and then becomes a single structure after another $200 \mu \mathrm{m}$. The width of the smallest feature in the image is roughly $4 \mu \mathrm{m}$, which is the diffraction limit of the system, suggesting that a finer structure could exist. In Fig. 3 the free-electron density is plotted at several lateral cross sections as labeled with numbers in Fig. 2(b). In view of the possible finer structure than the diffraction limit, it should be noted that the density of free electrons could be higher. For the electron densities measured it is possible that both multiphoton ionization and avalanche processes play a role. It requires further study to determine whether what we have observed is the initiation of filamentation, local breakdown, or perhaps an intermediate regime.

The holographic recording method is also capable of capturing time sequences of the plasma-generation process. We employ a setup similar to that depicted in Fig. 1, in which mirror M4 is replaced with a mirror array consisting of four mirror segments, each of which has independent controls for angular and axial displacements. The displacements of the mirror segments are carefully tuned such that the four reflected probe pulses propagate with a small relative time delay and angular separation. The four pulses spatially overlap in the region of the air discharge, although they are temporally separated. Therefore, each probe pulse samples the event at a time set by the displacement of the mirror. The CCD camera records the four holograms with magnification $M=2.5$ at $L=16 \mathrm{~cm}$, where the four probe beams (and thus the holograms) are spatially separated. The variable time delay line synchronizes the pump and probe pulses at the onset of the plasma. We set the separation angle between the probe pulses to be sufficiently small $\left(<3^{\circ}\right)$ that the events are recorded at approximately the same angle. However, to spatially separate the four holograms in the CCD sensor plane, the effective angular aperture of each individual hologram is limited to the separation angle between the probe pulses.
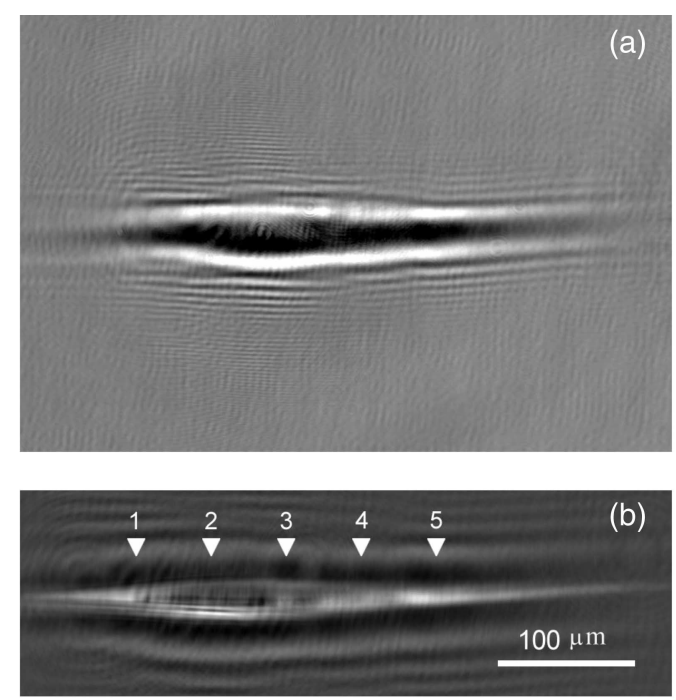

Fig. 2. (a) In-line hologram of the plasma distribution. The image resolution is $2184 \times 1472$, and the pixel size is $6.8 \mu \mathrm{m} \times 6.8 \mu \mathrm{m}$. The hologram is captured at $L=30 \mathrm{~cm}$ with a magnification of $M=12.5$. (b) Spatially resolved electron density calculated from the phase change.

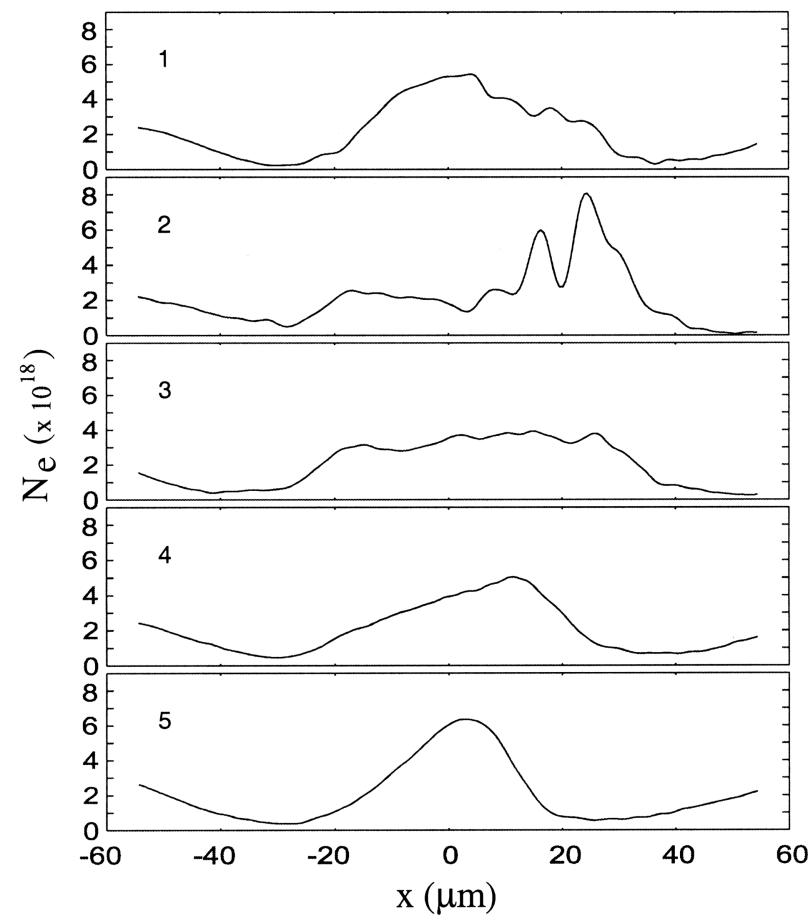

Fig. 3. Electron density distribution at various cross sections showing detailed plasma evolution. Distances from the onset of the plasma are $1,326 \mu \mathrm{m} ; 2,435 \mu \mathrm{m} ; 3$, $544 \mu \mathrm{m} ; 4,652 \mu \mathrm{m} ; 5,761 \mu \mathrm{m}$. 


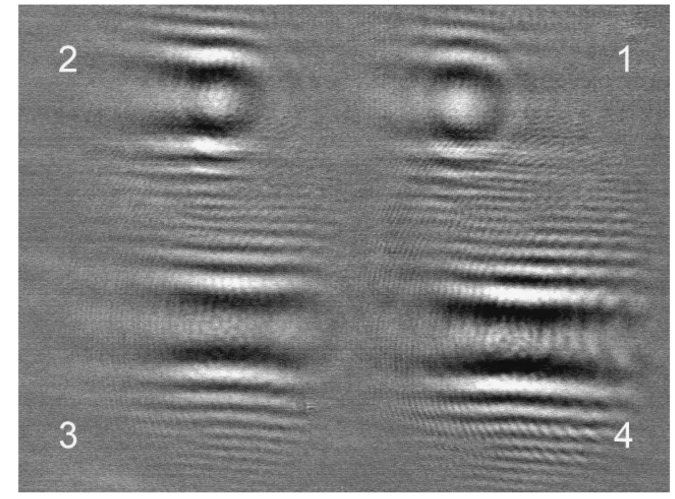

Fig. 4. Four instantaneous holographic snapshots of the plasma evolution. Image resolution, $640 \times 480$; pixel size, $10 \mu \mathrm{m} \times 10 \mu \mathrm{m} ; L=16 \mathrm{~cm} ; M=2.5$.
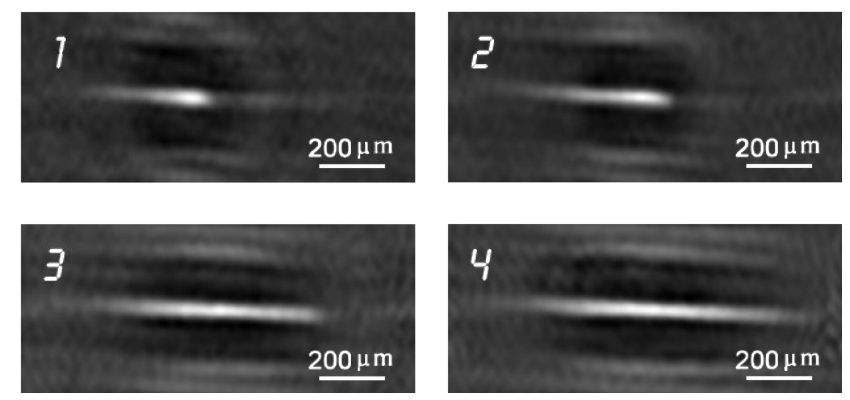

Fig. 5. Instantaneous time evolution of the electron density. The relative time of the snapshots is $t_{1}=0, t_{2}=1 \mathrm{ps}$, $t_{3}=2 \mathrm{ps}$, and $t_{4}=2.7 \mathrm{ps}$. Because of the small angular aperture, fine structure is not recovered.

Figure 4 shows the holograms recorded on the camera at times $t_{1}=0, t_{2}=1 \mathrm{ps}, t_{3}=2 \mathrm{ps}$, and $t_{4}=2.7 \mathrm{ps}$. The energy of the pump laser pulse is the same as in the previous experiment. We numerically reconstruct the holograms and calculate the density of free electrons through the reconstructed phase. The electron density distribution of the four events is shown in Fig. 5. Despite the reduced spatial resolution as a result of the limited angular aperture, this result gives a dynamic picture of the time evolution of the plasma density.

In summary, we have presented a holographic method of measuring the spatial distribution of free electrons generated at the trail of a femtosecond pulse propagating in air. Our method can also be used to measure plasma generated in other transparent materials (liquids, solids) and under different focusing conditions. We have also demonstrated the capability of our technique to capture a sequence of frames in a single experiment.

This work was supported by the Engineering Research Centers Program of the National Science Foundation under award EEC-9402726. M. Centurion's e-mail address is mcenturi@sunoptics.caltech.edu.

\section{References}

1. S. Schedin, G. Pedrini, H. Tiziani, A. Aggarwal, and M. Gusev, Appl. Opt. 40, 100 (2001).

2. Z. Liu, M. Centurion, G. Panotopoulos, J. Hong, and D. Psaltis, Opt. Lett. 27, 22 (2002).

3. R. Rokitski, P. Sun, and Y. Fainman, Opt. Lett. 26, 1125 (2001).

4. A. Bugayev and M. Gupta, Opt. Lett. 28, 1463 (2003).

5. L. Bergé and A. Couairon, Phys. Rev. Lett. 86, 1003 (2001).

6. S. Tzortzakis, L. Bergé, A. Couairon, M. Franco, B. Prade, and A. Mysyrowicz, Phys. Rev. Lett. 86, 5470 (2001).

7. M. Mlejnek, M. Kolesik, J. V. Moloney, and E. M. Wright, Phys. Rev. Lett. 83, 2938 (1999).

8. H. Schillinger and R. Sauerbrey, Appl. Phys. B 68, 753 (1999).

9. S. Tzortzakis, M. A. Franco, Y.-B. André, A. Chiron, B. Lamouroux, B. S. Prade, and A. Mysyrowicz, Phys. Rev. E 60, R3505 (1999).

10. S. Tzortzakis, B. Prade, M. Franco, and A. Mysyrowicz, Opt. Commun. 181, 123 (2000).

11. A. Talebpour, S. Petit, and S. L. Chin, Opt. Commun. 171, 285 (1999).

12. A. Braun, G. Korn, X. Liu, D. Du, J. Squier, and G. Mourou, Opt. Lett. 20, 73 (1995).

13. Y. Silberger, Opt. Lett. 15, 1282 (1990).

14. P. Monot, T. Auguste, L. A. Lompré, G. Mainfray, and C. Manus, J. Opt. Soc. Am. B 9, 1579 (1992).

15. D. Gabor, Nature 161, 777 (1948).

16. H. J. Kreuzer, M. H. Jericho, I. A. Meinertzhagen, and W. Xu, J. Phys. Condens. Matter 13, 10729 (2001).

17. R. W. Boyd, Nonlinear Optics, 2nd ed. (Academic, San Diego, Calif., 2003). 